Copyright ( 2013 IEEE. Personal use of this material is permitted. Permission from IEEE must be obtained for all other uses, in any current or future media, including reprinting/republishing this material for advertising or promotional purposes, creating new collective works, for resale or redistribution to servers or lists, or reuse of any copyrighted component of this work in other works. 


\title{
Implementing Intelligent Pedagogical Agents in Virtual Worlds: Tutoring Natural Science Experiments in OpenWonderland
}

\author{
Mohamed Soliman and Christian Guetl \\ Institute for Information Systems and Computer Media \\ Graz University of Technology \\ Graz, Austria \\ Muhamed.soliman@gmail.com, Christian.Guetl@iicm.TUGraz.at
}

\begin{abstract}
Intelligent Pedagogical Agents (IPAs) can be thought of as embodied intelligent agents that are designed for pedagogical purposes to support learning. They can be designed in particular for virtual worlds. Virtual worlds are becoming an interesting medium for engineering education for the properties of visual collaboration abilities providing authentic learning experiences and for the opportunity of providing active learning. However, virtual worlds need more educational support to be more inhabited with increased learning services. Incorporating intelligent pedagogical agents into virtual worlds adds such learning support by adding intelligence, improving believability, and the opportunity to increase communication with an artificial educator. However the implementation of intelligent pedagogical agents and adopting them in a virtual world require several efforts with different aspects of implementation. This paper reports our first prototype implementation of an IPA interacting with a learner and a learning object in natural science experiment in a virtual world while providing supporting multimodal communication abilities. The IPA has features of text chat based on the Artificial Intelligence Markup Language (AIML), a text-to-speech synthesis function, and non-verbal communication abilities through gesture animation. The implementation is presented through explained scenarios of the IPA tutoring an experiment or monitoring a learner avatar interaction with a learning object in a Virtual World. The IPA \& the learning scenarios are implemented in the open source of Open Wonderland.
\end{abstract}

Keywords-Intelligent Pedagogical Agents; Virtual Worlds; Intelligent Agents

\section{INTRODUCTION}

In a virtual world, users are represented by avatars enjoying collaboration abilities, immersion, and being able to run visual simulations of objects. The impact of utilizing a virtual world for learning includes removing time and distance barriers to collaborate with others visually, [1]. STEM (Science, Technology, Engineering, and Mathematics) education has current attempts to adopt virtual worlds for learning in several educational institutions, [2]. This gives the opportunity for online labs and engineering education extension.

With the advent of utilizing advancements in ICT and Internet, experiments can be conducted remotely. This has been seen in the area of remote and online labs and simulation to open the opportunity for remote users to get chances of learning and experimentation with expensive or remote settings. Thus it is to take advantage of scalability, costeffectiveness, availability of the resources 24 hours, and utilizing other ICT innovations. One example is the collaboration among remote users who can be in different places simultaneously but not hindered to experimentation activities. An added value to remote experimentation is adding rich visualization to be able to visualize the elements and the control of the experiment and even add computerized visualization of the result (such as visualizing a magnetic field) that is not possible in reality. Also, adding collaboration support. Therefore, the use of virtual worlds for engineering education in performing remote experimentation is seen as an added value as it gives 3D visualization, collaboration support, and the potential for adding contextualization [4]. Lila project [3] is an example of online labs efforts that adopts Open Wonderland as a virtual world. Lila is a library of virtual and remote labs as a consortium of eight universities and three companies.

However, remote instruction support is still needed for online labs. This instruction support should also comply with the potential of increased remote learners who are represented by avatars in the virtual world. An instructor in a virtual world is also an avatar that can provide such support. That is not enough, given the potential increase in the number of users and the potential intelligent and pedagogical automated support possible by ICT innovations as well. This mandates computerized support to the remote experiment utilizing ICT innovations not only for conducting it but providing autonomous pedagogical support to the learner users (avatars).

There is a lack of autonomous support in virtual worlds. For example, for learners who don't get peers find themselves alone with lack of guidance. Unless there are educator avatars available for support, learners don't get enough support and suffer from lack of expert guidance. Another problem is the need to feel that the environment is being inhabited. Otherwise, the learner feels bored and reluctant to learn. Furthermore, there is always pedagogical tactics that ask for more engagement in the classroom should also be in the virtual world so as to get motivated and engaged in completing the 
required tasks. In general, it is by adopting methods for managing the environment towards immersive and autonomous learning support.

One method of improving the pedagogical support and adding pedagogical intelligence is the adoption on intelligent pedagogical agents (IPAs) who can play the roles of teachers or peers. They can be available $24 / 7$ to provide instructional functions for immersed learners taking advantages of what ICT abilities can bring and utilize it in the service for educating the learners.

We have been investigating intelligent pedagogical agents for learning finding several benefits to learning, see [5], [6], [7]. IPAs add pro-activeness, interaction, engagement of learners in a virtual learning environment. In particular to 3D virtual worlds that offer the authentic learning experiences with rich visualization combined with collaboration. In particular implementation, Open Wonderland [8] is an open source Java based virtual world that has been used for providing physics experiments in [9] and more. However, virtual worlds need intelligence abilities and learning support. Pedagogical agents can be central points of interaction and learning service providers for learners in those environments adding to believability of the environment and further engagement while providing learning support. Design and implementation of intelligent pedagogical agents also entail investigating intelligent agents and their frameworks from AI research as well. Hence, we reported initial implementation results of a learning scenario based on the Belief-Desire-Intention (BDI) model while evaluating JACK and Jadex frameworks in [10]. The intelligent agent part can be a basis for the design and development of IPAs providing interesting intelligent behavior such as autonomy, goal-directed behavior as well as re-activeness while integrating with pedagogical plans [10]. However, integration and adoption in the virtual world is needed.

In this paper, we report our results on implementing learning scenarios with intelligent pedagogical agent in Open Wonderland virtual world. This includes integrating the intelligent agent framework with the Open Wonderland environment, adopting visual behavior of a Non Player Character (NPC) module while controlling gestures, integration of multi-modal communication module, and controlling (tutoring) a physics experiment. The multi-modal communication module integrates gesture generation, text chat (through Natural Language question making), and Text-ToSpeech generation. The pedagogical agent mediates interaction between the learning object and the avatar, extending the work in [9] to either allow tutorials or to monitor learner interaction in the 3D environment.

This paper is organized as follows. Section 2 provides background and related work of virtual worlds for learning and adopting intelligent pedagogical agents. Section 3 provides the requirements and the design of an IPA in the Open Wonderland environment explaining features of internal, external modules adopted, and developed ones. Section 4 discusses scenarios of learning with the implemented intelligent pedagogical agent in Open Wonderland. Section 5 concludes and provides future work and limitations.

\section{BACKGROUND AND RELATED WORK}

Several projects exist that adopts learning in the immersive virtual learning environment such as Project Virtual Singapura [11] and Rivercity [12]. [13] utilizes the collaborative abilities of the Open Wonderland virtual world environment to support entrepreneurs in a virtual incubator world. Efforts reported in [14], [9], and [13] adopt Open Wonderland virtual world environment to extend physics experiments to virtual world environments thus allowing collaborative abilities and more.

Open Wonderland is an Open source java based virtual world environment [8]. While Open wonderland is used for vast purposes such as gaming, collaborative business applications, it is an open source that allows changes to be used for education purposes.

Providing narrative and dialogue functions increase engagement and immersion in the environment. [15] reported increase in the sense of presence as a result of utilizing narrative characters in the environment. [16] reported that animated pedagogical agents play a powerful motivational role and reported the persona effect as adding a lifelike character increase learner perception and performance [16]. Furthermore, adding conversation abilities to the pedagogical agent enables better interaction with the learner, [17]. For years, there has been active research in adopting intelligent pedagogical agents such as in [18][17][19][15][20][16].

Interaction with the learning object is important to the learner and the IPA since the role of the IPA is to explain the object and mandate a pedagogical aware module. One role of the pedagogical agent is to explain the object from the learning perspective to the learner. [21] provides a taxonomy of the learning object to be static, automated, or interactive while the importance of object interaction in the virtual world is reported in [22]. The IPA requires interacting with objects that are machine controllable so as to be able to present its animation, step through it, and explain its behavior. The object of interest can be a simulation of a machine, a physics experiment, a remote laboratory device, or a control panel of a ship. STEVE, the pedagogical agent relies on VRIDES that simulates 3D object behavior in the environment and interacts with it to provide illustrations to the learners, [20]. The visual aspect is for the learner and the non visual aspects are for interaction, control of the device, assessment of the action. Our work in [10] provides intelligent agent reasoning and command of the learning object to provide specific behavior relevant to check for learner response in reaction.

Another important aspect is the IPA's ability to take intelligent decisions and reason about pedagogical actions to take in the virtual environment. This is to provide cognitive support to virtual human model such as found in [18]. Intelligent agent support approach to collaborative learning environments is provided in [23] and a survey of pedagogical functions of agents are provided in [5]. Furthermore, our prior works in [6] and [10] refer to BDI based simulation of a learning scenario that involves a pedagogical agent, a learner, and a learning object with JACK and Jadex agent platforms. The purpose is to simulate a similar situation in the virtual world. The agent role is to either provide step-wise guidance to 
the learner who is interacting with the learning object or to control the learning object for demonstration purposes while utilizing the learner belief base.

\section{REQUIREMENTS AND DESIGN}

In the context of this research, the requirements for an intelligent pedagogical agent in a virtual world are summarized to be:

- Multi-modal conversation abilities with the learner.

- Embodiment and Animation.

- Emotional Support.

- Sensing and affecting the environment, learning objects, and the learner.

- Decision abilities: reasoning about the situation and selection among possibilities.

- Being pedagogical aware providing learner management functions such as idle time management, providing intelligent support and guidance and aware of the learning activity to provide expert response.

- Act as a central point of interaction between the learner and the environment providing verbal and non-verbal support to the learner.

We have selected Open Wonderland 3D environment for our implementation, [8]. Several physics experiments are being undertaken to utilize the collaboration and rich visualization abilities of this virtual world environment such as in [9]. An IPA implementation in the environment leads to adding pedagogical and intelligent support.

The development of the pedagogical agent, rather than a simulation, requires embodiment of a character module to visually play the role of the pedagogical agent. Furthermore, the learner (avatar) to pedagogical agent interaction in the environment mandates a multi-modal communication module. The agent intelligent behavior should provide input to the IPA communication module, should interpret learner questions and respond to it, as well as providing gestures compatible with the learning situation.

A schematic diagram of the implementation is depicted in Figure 1. The implementation is based on Open Wonderland and uses external open source modules. In Open Wonderland, it uses the NPC module to represent the IPA embodiment and animation aspects. A text-chat module is developed to provide dialogue function and interfaced to external chat-bot support. The interface of the IPA is to an external Text-to-Speech synthesis server. Details of the implemented modules are described below.

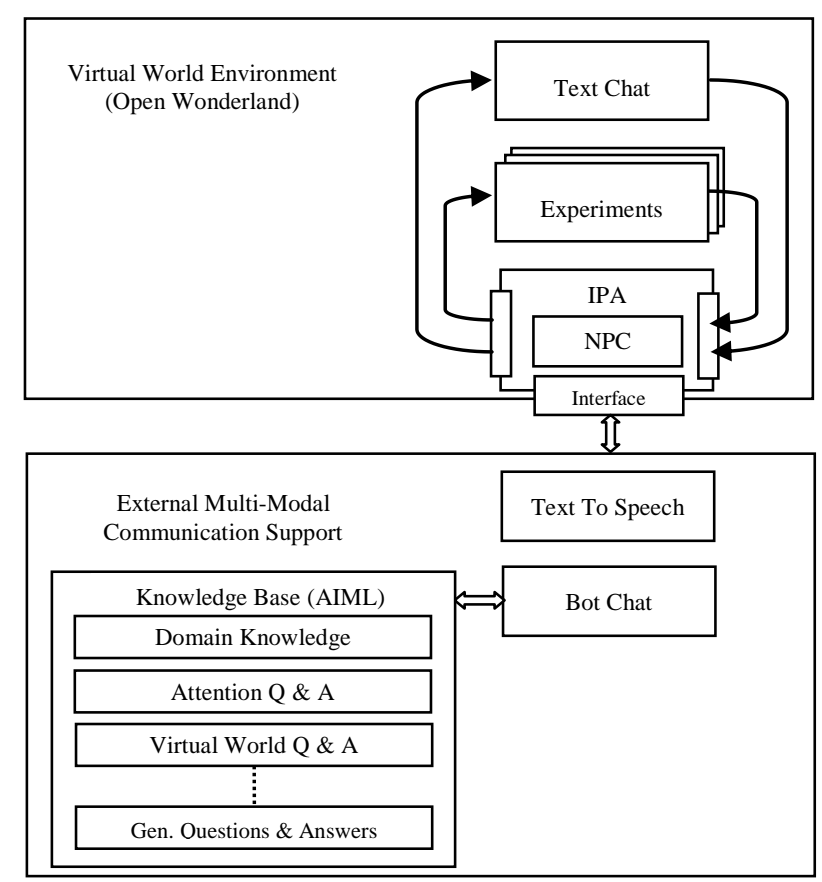

Figure 1. Schematic diagram of incoporating an intelligent pedagogical agent module in relation to submodules, externat modules, and Open Wonderland.

\section{A. Can the NPC Module Become the Virtual Teacher?}

NPC (Non Personal Character) is an available module in Open Wonderland that is a graphical representation similar to an avatar but with no user control, [8]. Therefore, it is appealing for utilization since it has several humanoid functions: appearance, moving, and providing gestures. However, it lakes decision functions. Therefore, the target is to develop on top of NPC module in Open Wonderland adding cognitive and decision abilities. This can be achieved by adopting it as an intelligent agent while the NPC takes the appearance and animation in Open Wonderland. Furthermore, the NPC module lacks conversation abilities.

A useful feature available with the NPC module is the proximity event listener that senses objects as becomes in proximity. When the learner gets into proximity of the IPA, the IPA knows and identifies the avatar and tries to initiate conversation and shows attention.

Providing gestures to the learner is important to support the emotional state and has influence on learning [16]. Therefore, adding this feature and for evaluation become important. Upon answering a question, the IPA provides a supporting gesture instead or with the text-based answer ${ }^{1}$. This is achieved by forwarding a request to the NPC based on the chat-bot answer to issue the gesture.

Since the NPC module is based on the avatar implementation, several avatar gestures are also available to the NPC as well according the Open Wonderland

${ }^{1}$ Under the redundancy principle, multiple simultaneous communication methods should be carefully handled [17]. I.e.

if text is used, a simultaneous voice is better prohibited. 
implementation, [8]. For example a "Yes" gesture is used to show approval and encouragement and the "No" gesture is used to show an occurring mistake and disapproval.

An interesting aspect is when the IPA senses avatar emotional state in Open Wonderland. An approach is to detect the avatar module gesture generation or in the work of [24] where a text chat module carryings Open-Wonderland compatible gestures through writing emoticons in the text chat module.

\section{B. Integrating an Intelligent Agent Paradigm with Open Wonderland}

Adding decision abilities to the pedagogical agent is enhanced by intelligent agent function to support reasoning based on the BDI model [10]. The task is challenging in different factors including the different threads operation of the virtual world and the agent platform. Furthermore, the vast events happening in the world can mandate selective (filtered) perception. The design of the perception module has three functions: 1) to sense the environment on events important to the agent 2) filter the events of interest 3) act in the environment with affecter. Therefore, the design of the interface between open wonderland and Jadex has two main components:

- A virtual world component that listens to the events of interest and filter them, dispatch the events through HTTP requests to Jadex agent

- A Jadex Interface agent that receives the requests, dispatch it to the corresponding agent and also managing effectors to the virtual world component, see Figure 2.

While the agent controlled operation to the IPA is ongoing, the interface component is achieved, a proof of concept is provided in [10] as the objective to provide intelligent-agent supporting reasoning to learning and bringing it into the 3D domain. The work in [10] simulates the same scenario focusing on the intelligent agent behavior and is implemented in two platforms: JACK [25] and Jadex [26].

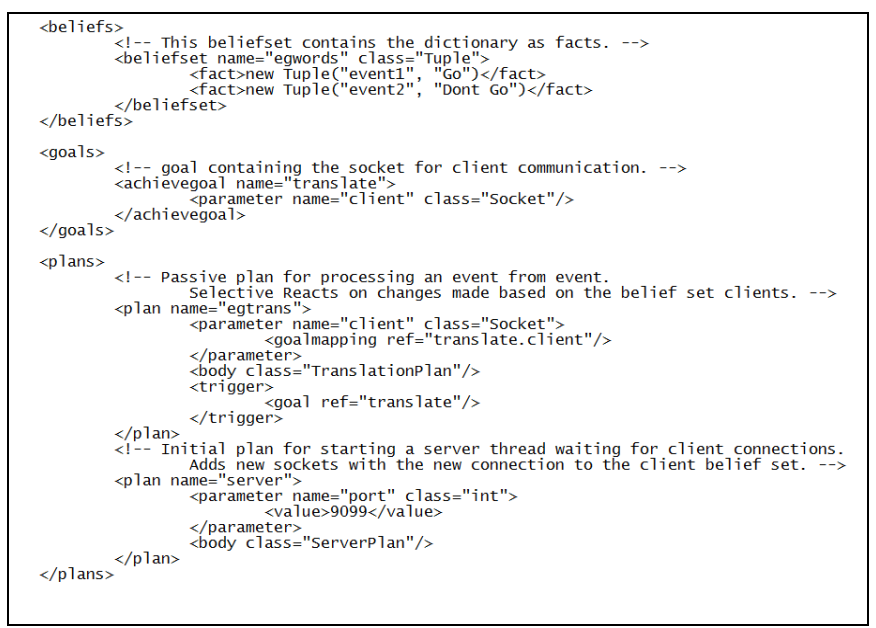

Figure 2. Snapshot from the Agent Definition File (ADF) representing an interface agent in Jadex.

\section{Text Based Chat Module}

To allow the learner-IPA interaction in text, a text-chat HUD (Head up display window) is developed in Open Wonderland. Text messages sent by the learner to the IPA are typed in this window and IPA text directed to the learner is displayed as well in this window. An example text is the IPA to welcome the learner upon starting a conversation.

In order to allow question and answer conversations, RebeccaAIML is employed for chat-bot language support [27]. The tool relies on the Artificial Intelligence Markup Language (AIML), an XML-compliant language to provide textual natural language processing of questions and answers to virtual characters, [28]. An example of the populated AIML file is shown in Figure 3. The text-chat module of the IPA is linked to RebeccaAIML that sends questions asked by the learner to its server that will send a reply back of the answer according to pre-defined knowledge base. Processing allows variations of the syntax so that to adapt to different names of the Bot, and variations of the questions.

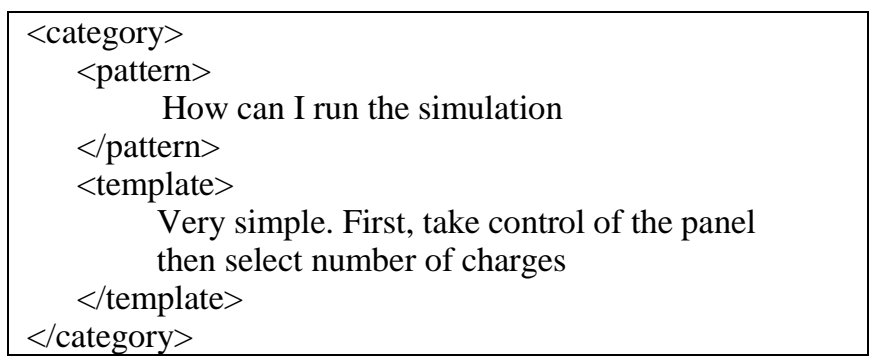

Figure 3. Sample AIML file containing a simple question and answer about the experiment.

The AIML based knowledge is populated by different types of questions and answers:

- Domain knowledge. For example capacitor knowledge base, answers about running the experiment, and level of difficulty of the experiment.

- Questions and answers about the environment. For example, what experiments are available? This is to allow the learner to obtain information about the environment.

- Attention and gesture supporting knowledge base to emotional state of the learner. For example, Can I help you? It is not a difficult experiment (linked to gesture).

\section{- General questions and answers.}

An important aspect in considering human question making is the possible big variations of the same question syntax. AIML specification allows extension to context, variation of the question pattern by synonyms, and wildcard usage.

AIML files can be added to the knowledge base by incorporating general or domain AIML files through the management tool of RebeccaAIML. Also new questions and answers can be added into the existing KB files. This feature is used in a training mode the conversation of the IPA to expand 
its conversation knowledge from Open Wonderland session to allow an educator or administrative user to customize the IPA.

\section{Adding Voice}

The voice feature is added through text to speech synthesis. Mary TTS is a java based open source Text-To-Speech (TTS) system that has been developed as a joint effort of the German Research Center for Artificial Intelligence (DFKI) and the Institute of Phonetics at Saarland University and is currently maintained in DFKI [29]. Mary TTS is client-server based allowing sending speech synthesis requests through the HTTP protocol to Mary server. Several other features of Mary TTS are provided in [29] [30]. An interesting feature is its ability to provide synthesis of emotional speech ${ }^{1}$. When it is needed that the IPA speaks in voice, it sends HTTP requests with the required text to be synthesized to Mary server which will return the speech in voice.

\section{E. Experiment Object and its Interaction}

An experiment has three aspects: control Panel, visualization of the result and its internal operations. The objective is to take an experiment module as a unit of learning that allows the pedagogical agent to manipulate it in learning interaction with the learner avatar. A Swing-based module is developed and deployed in Open Wonderland to represent the leaning object control panel and to provide necessary information useful for the IPA to provide step-wise guidance and to be able to assess the correct operation.

- Simulates experiment or device operation.

- Events are propagated to IPA upon avatar selecting a control and value. It is useful for the IPA to be notified upon learner interaction with the experiment.

- Ability to control the experiment internally by the IPA not only the user to provide tutorial.

- Ability to intervene the operation preventing the learner form incorrect operation of the experiment.

\section{LEARNING With A PEdAgogicAl AgENT IN A VIRTUAL WORLD: REALIZED SCENARIOS}

As shown, the objective is to extend experiments learning in the immersive virtual learning environment taking advantages such of its immersion, visualization, authentic learning experiences adding interactive learning support by means of a pedagogical agent. We have added a swing module that simulates a control panel of a learning object while there can be different ones such as in the research of [9] and [31]. With the above mentioned implementation, different forms of learning supported scenarios are realized as described below.

\section{A. Capturing Learner Attention upon Proximity}

While learner avatars roam in the virtual world, intelligent pedagogical agents should be available to offer help. In this

${ }^{1}$ Emotional speech synthesis is to alter the synthesized voice so as to represent an emotion. This feature is not implemented in this first prototype. prototype implementation, when the learner avatar gets into proximity of the IPA, the IPA will initiate conversation in attempt to capture the learner attention and engagement. A text-chat window will appear as a result of the proximity event.

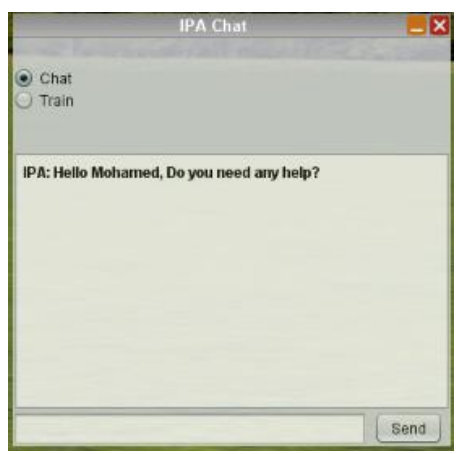

Figure 4. The text-chat window when the IPA initiates conversation as the learner avatar gets into proximity.

Then the IPA provides hints on being available for help ("What can I do for you" for example, "Would you like to see an experiment?")

Several extensions are possible to this feature by giving the IPA the opportunity to weave for example, or providing a list of available IPA's and their roles in support.

\section{B. Learner Conversation}

The learner has the option to ask questions such as "What experiments do you have?", knowledge questions such as "What is a capacitor?". The IPA will answer those questions based on the knowledge base available. Questions can be recognized to provide simple emotional support through gestures. When the IPA receives the question, "is it an easy experiment", the answer with "yes" in either voice or text is accompanied with yes gesture and the opposite with no. Figure 5. shows a dialogue between the learner and the IPA.

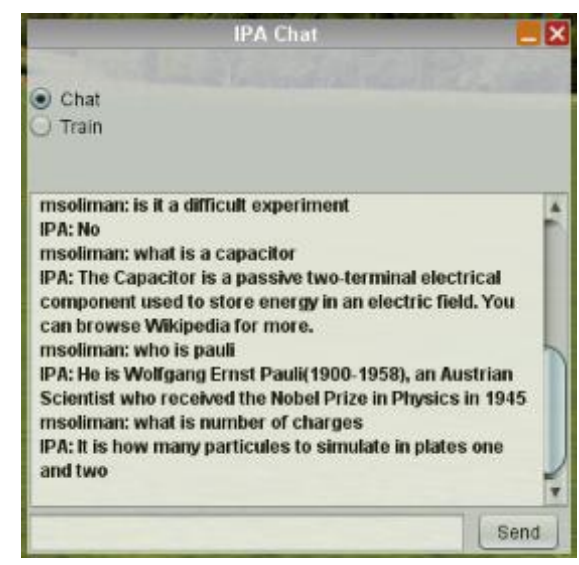

Figure 5. A text chat window showing a dialogue between the user and the IPA 
The Learner can also ask requests such as "Can you show capacitor experiment". This question is interpreted as a request to instantiate a learning activity of "Capacitor learning", for example. The IPA will invoke the Open Wonderland learning Object (in our case is a simulation of a swing panel). After invoking the simulation experiment, the learner can further ask questions and provide requests to the IPA. Figure 2 shows learner interaction with a pedagogical agent in a learning setting for a capacitor simulation experiment. The learner asks questions to the pedagogical agent or asks for providing a tutorial (in text or voice). The actual capacitor simulation module contains a visualization of particles animation.

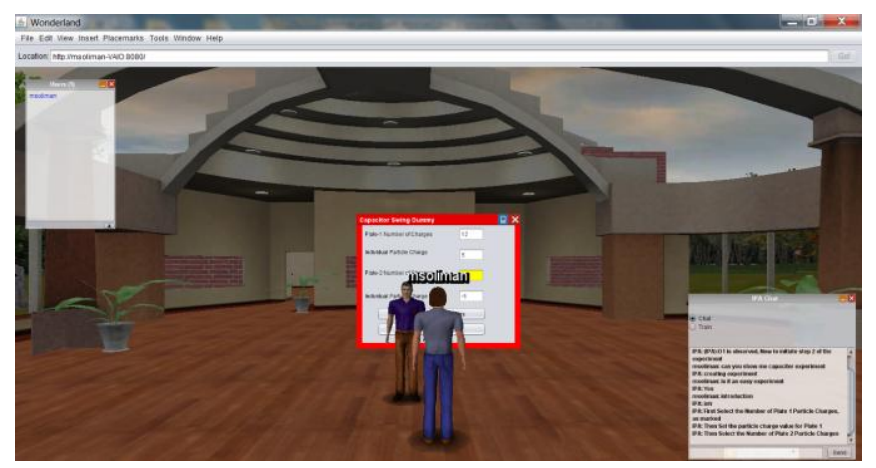

Figure 6. A Pedagogical Agent providing a tutorial on a capacitor experiment in OpenWonderland

\section{Tutoring the Experiment}

In addition to questions about the experiment mentioned above, the IPA provides a stepwise tutorial on the experiment by sequencing on the panel entry control and explains the purpose of each field with supporting information, see Figure 7. This is conducted in either text or speech while visually highlighting the selected field to the learner.

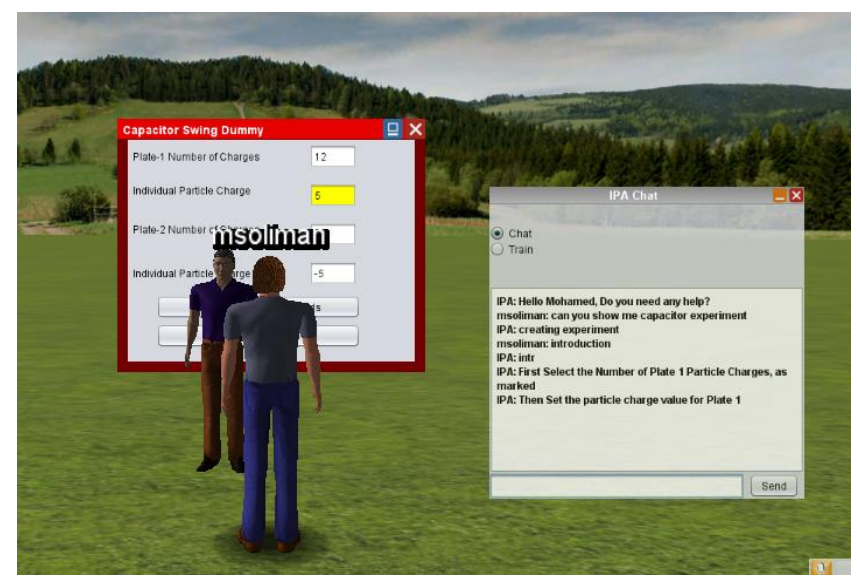

Figure 7. A Pedagogical agent tutoring an experiment while highlighting the corresponding field in the panel.

\section{Monitoring Learner interaction with an Experiment}

An important function of the IPA is to monitor the interaction with the learning object so as to ensure correct operation and correct learner interaction with the experiment. The IPA provides two types of feedback, correcting feedback and supporting feedback. When the interaction is correct, the IPA can provide, in text or speech, a supporting feedback to "continue" supported by a positive gesture. Updating learner data can occur in conjunction with this scenario to reflect his/her performance. Figure 3 shows the pedagogical agent monitoring learner interaction with the learning module to check for correct operation and providing feedback to the learner avatar. Several additional features include managing idle time of the learner avatar, providing further attentive gestures, or controlling parts of the experiment though natural language by the learner avatar.

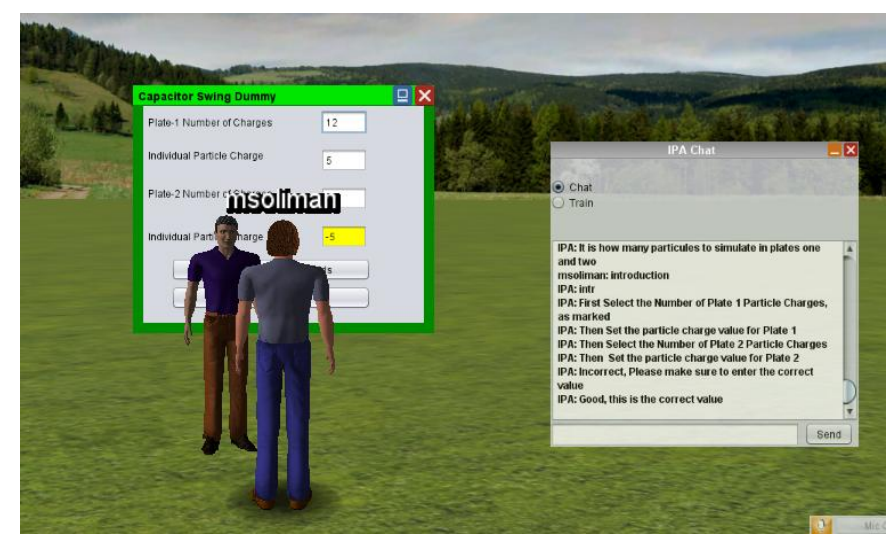

Figure 8. A pedagogical agent monitors learner interaction with the experiment providing feedback.

\section{E. Training the IPA}

With the facility of updating the knowledge base of the IPA, the user provides questions and the corresponding answers with the train mode selected. This has two purposes. First, for educator avatars to update the knowledge base of the IPA to be able to support different experiments (different domain knowledge). Second, is for the learner to be able to teach the IPA. The later feature falls in the category of teachable agents, [5]. In the first case, it will be considered as "reliable knowledge" while in the later, it can be considered as "unreliable knowledge" that can be used for research purposes of an agent representing a student but with further considerations.

\section{CONCLUSION AND FURTHER CONSIDERATIONS}

The paper discusses learning scenario first prototype implementation of intelligent pedagogical agents (IPAs) in the virtual world of Open Wonderland. Our focus is fostering learning with experiments in the environment by means of the IPA. The IPA chats with the learner to answer questions, provides demonstrations, and gives emotional support. The objective is to autonomously support the learner in the immersive environment. In order to realize those features along 
with desired reasoning abilities, an NPC module in the Open Source Wonderland has been utilized with adding features in addition to supporting its decision abilities by interfacing with an intelligent agent platform.

A multi-modal communication module is central to the IPA since IPA is the focal point of interaction with the learner and helps in improving engagement, believability, and more. Question answering through a text chat feature is implemented by adopting the Artificial Intelligent Markup Language (AIML). It also allows training the pedagogical agent to update the question/answer knowledge base. Converting to voice is enabled by a Text to Speech Engine. The IPA aware of knowledge of the learning experiment in addition to be able to communicate with the learner provides an unconventional user interface

With this implementation, the avatar learner can ask the pedagogical agent questions on the scope of an experiment, gets information about the experiment, listens to and sees a demonstration to running an experiment visually. The IPA has the ability to monitor learner actions versus expected ones to run the experiment and upon errors, the IPA provides feedback, corrects errors, and updates learner assessment data.

Further considerations are ongoing including operation and agent management providing environment reasoning and awareness, pedagogical and learning awareness, and extending the scenarios of interaction with the learning object to allow collaborative work.

\section{REFERENCES}

[1] C. Guetl, "The Support of Virtual 3D Worlds for Enhancing Collaboration in Learning Settings, " in F. Pozzi and D. Persico (Eds.), Techniques for Fostering Collaboration in Online Learning Communities: Theoretical and Practical Perspectives. IGI Global. 2011).

[2] Immersive Education, http://immersiveeducation.org/, Online, Accessed Dec. 3, 2012.

[3] LiLa: Library of Labs, http://www.lila-project.org/home.html, Online, Accessed Dec. 3, 2012.

[4] T. Machet, D. Lowe, and C. Guetl, "On the Potential for Using Immersive Virtual Environments to Support Laboratory Experiment Contextualisation," European Journal of Engineering Education, Vol. 37, No. 6, December 2012, 527-540.

[5] M. Soliman and C. Guetl, "A Survey of Pedagogical Functions of Intelligent Agents in Virtual Learning Environments," Journal of Internet Technology (JIT), Special Issue on Agent Technology in Cyberspace, Vol 12, No 6, 2011.

[6] M. Soliman and C. Guetl, "Evaluation of Intelligent Agent Frameworks for Human Learning in Virtual Worlds," International Journal of Engineering Pedagogy (iJEP), Vol 1, No 3, 2011.

[7] M. Soliman and C. Guetl, "Review and Perspectives on Intelligent Multi-Agent Systems' Support for Group Learning," World Conference on Educational Multimedia, Hypermedia \& Telecommunications, EDMEDIA 2010, June 2010, Toronto, Canada.

[8] OpenWonderland, http://openwonderland.org/, Online, Accessed Oct. 22, 2012.

[9] B. Scheucher, P. Bailey, C. Guetl, and V. J. Harward, "Collaborative Virtual 3D Environment for Internet-accessible Physics Experiments," International Journal of Online Engineering (iJOE), Vol. 5, pp. 65-71, 2009.

[10] M. Soliman and C. Guetl, "Experiences with BDI-Based Design and Implementation of Intelligent Pedagogical Agents," International Conference on Interactive Collaborative Learning, ICL 2012, Sept 2628, Villach, Austria.
[11] Project Virtual Singapura, https://www.futureworld.sg/projects/virtualsingapura, Online, Accessed Dec. 3, 2012.

[12] River City Project, http://muve.gse.harvard.edu/rivercityproject/ , Online, Accessed Dec. 3, 2012.

[13] C. Guetl and J. Pirker, "Implementation and Evaluation of a Collaborative Learning, Training and Networking Environment for Start-Up Entrepreneurs in Virtual 3D Worlds," International Conference on Interactive Collaborative Learning, ICL 2011.

[14] C. Guetl, T. Scheucher, P. H. Bailey, J. Belcher, F. R. dos Santos, and S. Berger "Towards an Immersive Virtual Environment for Physics Experiments Supporting Collaborative Settings in Higher Education," In A. Azad, M. Auer, and V. J. Harward (Eds.), Internet Accessible Remote Laboratories: Scalable E-Learning Tools for Engineering and Science Disciplines. IGI Global. 2012.

[15] S. McQuiggan, J. Rowe, and J. Lester, "The Effects of Empathetic Virtual Characters on Presence in Narrative-centered Learning Environments," In Proceedings of SIGCHI Conference on Human Factors in Computing Systems, CHI'08, 2008.

[16] J. Lester, S. Converse, S. Kahler, T. Barlow, B. Stone, and R. Bhogal, R. "The Persona Effect: Affective Impact of Animated Pedagogical Agents," In Proceedings of CHI '97 (pp. 359-366), Atlanta, March 1997, Miami, Florida, USA.

[17] B. Weusijana, R. Kumar, and C. Rose, "MultiTalker: Building Conversational Agents in Second Life using Basilica, Second Life," Education Community Convention, Purple Strand: Educational Tools and Products, 2008, Tampa, FL.

[18] J. Funge, X. Tu, and D. Terzopoulos, ”Cognitive Modeling: Knowledge, Reasoning, and Planning for Intelligent Characters," Proceedings of SIGGRAPH'99: 26th Conference on Computer Graphics (pp. 29-38)., 1999. New York: ACM Press.

[19] M. Soliman and C. Guetl, "Intelligent Pedagogical Agents in Immersive Virtual Learning Environments: A review," Proceedings of 33rd International Convention on Information and Communication Technology, Electronics and Microelectronics, MIPRO, May 24-28 2010, Opatija, Croatia.

[20] W. Johnson, J. Rickel, R. Stiles, and A. Munro, A. (1998), "Integrating Pedagogical Agents into Virtual Environments," Teleoperators and Virtual Environments. Vol. 7(6), pp. 523-546. 1998.

[21] A. Schmeil and M. Eppler, "Formalizing and Promoting Collaboration in 3D Virtual Environments - A Blueprint for the Creation of Group Interaction Patterns," In Proceedings of First International Conference of Facets of Virtual Environments (FaVE 2009), July 27-29, 2009, Berlin, Germany.

[22] P. Jorissen and W. Lamotte "A Framework Supporting General Object Interactions for Dynamic Virtual Worlds, "In Proceedings of the 4th International Symposium, SG 2004, Banff, Canada, May 23-25, 2004.

[23] M. Soliman and C. Guetl, "Review and Perspectives on Intelligent Multi-agent Systems' Support for Group Learning," World Conference on Educational Multimedia, Hypermedia \& Telecommunications EDMEDIA 2010, June 2010, Toronto, Canada.

[24] S. Amarakeerthi, R. Ranaweera, M. Cohen, and N. Nagel, "Mapping Selected Emotions to Avatar Gesture," In IWAC: 1st Int. Workshop on Aware Computing, Japan Society for Fuzzy Theory and Intelligent Informatics. Sep. 2009, Aizu-Wakamatsu.

[25] JACK, http://aosgrp.com/products/jack/index.html , Online, Accessed Dec.3, 2012.

[26] Jadex, http://jadex-agents.informatik.uni-hamburg.de, Online, Accessed Dec. 3, 2012.

[27] Rebecca AIML, http://rebecca-aiml.sourceforge.net/, Online, Accessed Dec. 3, 2012.

[28] AIML: Artificial Intelligence Markup Language, http://www.alicebot.org/aiml.html, Online, Accessed Dec. 3, 2012.

[29] Mary Text To Speech, http://mary.dfki.de/, Online, Accessed Dec. 3, 2012.

[30] M. Schröder and J. Trouvain, "The German Text-to-Speech Synthesis System MARY: A Tool for Research, Development and Teaching," International Journal of Speech Technology, 6, pp. 365-377, 2003. Available online at 
http://mary.dfki.de/documentation/publications/schroeder trouvain2003. pdf

[31] J. Pirker, S. Berger, C. Guetl, J. Belcher, and P. Bailey, "Understanding Physical Concepts using an Immersive Virtual Learning," In
Proceedings of 2nd European Immersive Education Summit, 26-27 Nov. 2012, Paris.

[32] J. Kaplan and N. Yankelovich, "Open Wonderland: An Extensible Virtual World Architecture,” IEEE Internet Computing, 2011, 38-45. 